\title{
Go $\begin{array}{ll}\text { Research Square } & \text { They should not be considered conclusive, used to inform clinical practice, } \\ \text { or referenced by the media as validated information. }\end{array}$
}

\section{Computer Modeling of Carotid Endarterectomy With the Different Shape Patches and Prediction of the Atherosclerotic}

\section{Vladimir Geraldovich Borisov}

Kemerovo State University https://orcid.org/0000-0003-1037-5976

\section{Yuri Nikolaevich Zakharov}

Institute of Computational Technologies SB RAS https://orcid.org/0000-0002-8141-7873

Anton Nilolaevich Kazantsev

Kuzbass Cardiology Center https://orcid.org/0000-0002-1115-609X

Alexander Vladimirovich Korotkikh ( $\nabla$ ssemioo@rambler.ru )

Cardiac Surgery Clinic of the Amur State Medical Academy https://orcid.org/0000-0002-9709-1097

Yuri Ivanovich Shokin

Institute of Computational Technologies SB RAS https://orcid.org/0000-0002-8177-6294

Alexei Valer'evich Evtushenko

Kuzbass Cardiology Center https://orcid.org/0000-0002-2955-5689

\section{Leonid Semenovich Barbarash}

Kuzbass Cardiology Center https://orcid.org/0000-0001-6981-9661

Pavel Sergeevich Onishchenko

Kuzbass Cardiology Center https://orcid.org/0000-0003-0648-2584

Kirill Yur'evich Klyshnikov

Kuzbass Cardiology Center https://orcid.org/0000-0002-7232-5168

Evgeniy A. Ovcharenko

Kuzbass Cardiology Center https://orcid.org/0000-0003-2769-0278

\section{Research Article}

Keywords: carotid endarterectomy, computational fluid dynamics, wall shear stress, patch configuration

Posted Date: January 6th, 2022

DOI: https://doi.org/10.21203/rs.3.rs-1225320/v1

License: (1) (1) This work is licensed under a Creative Commons Attribution 4.0 International License.

Read Full License 


\section{Abstract}

Objective: The article describes a method for constructing geometric models of the carotid bifurcation and computer simulation of endarterectomy surgery with the patches of various configurations. The purpose of this work is to identify the areas of the greatest risk of restenosis in the constructed models and to conduct a comparative analysis of risk factors when using the patches of different widths and shapes.

Methods: The method is demonstrated on a reconstructed model of a healthy vessel. Its building is based on a preoperative computed tomography study of a particular patient's affected vessel. The flow in the vessel is simulated by computational fluid dynamics using data from the patient's ultrasound Doppler velocimetry. Risk factors are assessed through the hemodynamic indices on the vessel wall associated with Wall Shear Stress.

Results: The distribution of risk zones in the healthy vessel, presumably leading to its observed lesion (plaque), is analyzed. Comparative evaluation of 10 various patches implantation results is carried out and the optimal variant is determined. The proposed method can be used to predict the hemodynamic results of surgery using patches of various sizes and shapes.

\section{Introduction}

According to statistics, an acute cerebrovascular accident is one of the main causes of fatalities and long-term disabilities [1]. The stenosis of carotid arteries makes $20-30 \%$ of such disorders approximately [2]. The cause of stenosis is the deposition and growth of atherosclerotic plaque, which gradually blocks the lumen of the vessel. When significant stenosis (more than $75 \%-80 \%$ ) occurs, carotid endarterectomy (CEA) is indicated. The atherosclerotic plaque is removed there through a longitudinal incision of the vessel wall. Then, the incision is sutured either directly or with the implantation of a patch of a certain width and shape. The result of CEA is a local change in the geometric shape of the vessel, leading to a change in the flow hemodynamics. Studies of blood flow hemodynamic characteristics on a vessel wall are extremely difficult in vitro, and even more so in vivo. Therefore, the CFD (Computational Fluid Dynamics) methods are widely used to evaluate hemodynamic effects in vessels of a personal-specific form $[3,4]$.

The connection of atherosclerotic phenomena in blood vessels with such hemodynamic index as WSS (Wall Shear Stress) has long been known $[5,6]$. It is generally recognized that the risk of atherosclerosis is increased in the areas of low WSS values, where the oscillating flow also takes place [7 9]. Particularly, the risk zones include sections of the vessel wall where the recirculation or branching of the flow occurs in its vicinity $[10,11]$. The WSS index is a non-uniform tangent vector field defined on the inner vessel wall. This field dynamically changes in intensity and direction, which makes it difficult to evaluate its effect on the processes occurring on the vessel wall. Therefore, the integral indices associated with WSS are used. For example, the Oscillatory Shear Index (OSI) was proposed to describe the oscillatory behavior 
of WSS [8]. Together with OSI, the TAWSS index (Time Averaged WSS) is widely used. It characterizes the WSS value averaged over one or several cardiac cycles [12 14]. It is known [15] that there is a strong negative correlation between OSI and the magnitude of TAWSS. Therefore, the RRT (Relative Residence Time) index is often used. It is algebraically expressed in terms of OSI and TAWSS [16 18]. The RRT index gives an estimate of the average time spent by the blood particles and chemicals in certain near-wall areas [17]. At the same time, the TAWSS, being a vector field, contains information about the direction and speed of the chemicals and blood particles movement near the vessel wall [19]. Some other indices are also used to describe hemodynamic characteristics (see [10], [20 22]). The interdependence between different hemodynamic indices was investigated in $[23,24]$.

There are several papers, discussing various methods of performing CEA where hemodynamic indices are used to assess the risk of restenosis, e.g. [25 27]. The Comparison of CEA results performed both with and without patch implantation was presented in $[16,28,29]$. The comparative analysis of the distribution of the hemodynamic indices in patient-specific models of carotid bifurcation was performed in $[12,21,30]$. However, there are currently no generally accepted standards and recommendations for choosing the technique of surgery (with or without a patch) and the width or shape of the patch; this choice is left to the practicing surgeon and there is no consensus on this issue [26].

This paper describes a method for computer imitation of a CEA surgery with patches of various configurations. The method includes:

- reconstruction of a healthy vessel geometric shape based on preoperative data of the affected one;

- - creating geometric models that imitate CEA variants with the patches of different widths and shapes;

- analysis of hemodynamic indices in the constructed models using CFD.

The purpose of this work is to identify the areas of the greatest risk of restenosis in the constructed models and to conduct a comparative analysis of risk factors when using patches of different widths and shapes. The results of this analysis make it possible to select the patch form that is optimal in terms of minimizing the restenosis risk. In this paper, the proposed method is demonstrated in the case of the virtual implantation of 10 patches of various shapes into a vessel model. Its building is based on personally specific CTA (Computed Tomography Angiography) data.

\section{Materials And Methods}

\section{Source data for mathematical modeling}

The original geometric model of the vessel was reconstructed being based on a preoperative CTA examination of the affected left carotid artery of a particular patient. Fig. 1 (a) presents the flow area in the affected part of the vessel (the carotid artery is in the foreground). The curve segment in Fig. 1 (a) 
shows the supposed vessel wall position under the atherosclerotic plaque. The dotted line marks the plaque inner surface in the projection shown.

The source data for the flow simulation are the patient's postoperative UDV (Ultrasound Doppler Velocimetry) results. The dependence of the inlet flow rate on time is based on the data from the UDV study of the CCA (Common Carotid Artery). The ratio of flows through ICA (Internal Carotid Artery) and ECA (External Carotid Artery) was calculated using their cross-sectional areas and TAPV (Time-Averaged Peak Velocity). They were also obtained from the UDV data.

\section{Construction of geometric models}

The reconstructed three-dimensional model of a healthy vessel was built using SimVascular [31] and Salome [32] software. SimVascular was used to build vessel segments (see Fig. 1 (b-d)). Using a Python script of our design, these segments were later imported into Salome to build a geometric model and computational meshes. The reconstructed three-dimensional vessel model is shown in Fig. 1 (e). Further, this model is referred to as the base one and is denoted as m0. The black line on the vessel wall in Fig. 1 (e) indicates the incision line for the subsequent imitation of patch implantation.

The mentioned script was also used to visually simulate the result of a CEA surgery. It visually helps to draw the incision line and patch outlines on the vessel segmentation contours (Fig. 2). The information about the drawn lines is exported by the script to a data file as a list of the patch width values at its intersections with the segmentation contours. The script then uses this data to change the base model geometric shape, simulating the result of the patch implantation.

The modified models are the results of a virtual CEA. They were built by increasing (or decreasing) the perimeters of all segments of the $\mathrm{m} 0$ model that intersect with the incision line. This made it possible to simulate any form of patch or incision closure without a patch. Changing the perimeters was achieved by scaling segments relative to their geometric centers following the values recorded in the data file. By default, the scaling coefficient is assumed to be equal to the value of the relative increment of the perimeter stored in the file, divided by $2 \pi$. This choice is quite accurate for segments close in shape to ellipses with a small eccentricity. The scaling coefficient can be desirably adjusted manually for irregular segments. As a result of scaling, the upper (in Fig. 2) parts of the ICA proximal contours shifted up and began to intersect with the proximal contours of the ECA (not shown in Fig. 2). To correct this, one performed a small parallel displacement of all the ECA contours along the larger axis of the CCA distal contour. After that, a geometric model of the vessel and computational meshes on it were built. The meshes were then exported to the OpenFoam [33] for numerical calculations.

For comparative analysis, geometric models $\mathrm{m} 1-\mathrm{m} 10$ were constructed using the method described above. The m1-m9 models simulate the results of CEA surgery on the $\mathrm{m} 0$ model with the implantation of p1-p9 patches, respectively (Fig. 3). The m10 model (not shown in Fig. 3) simulates the suture of the incision without patch implantation. 
The data on the shapes of the patches are given in Table 1, which contains the width s of the patches in their cross-sections along the incision line. Point 0 of the column "Distance..." corresponds to the proximal end of the patch, point 3.9 does to the distal one. The incision line (shown in Fig. 1 (e)) was the same for all the models. Both its length of $3.9 \mathrm{~cm}$ and its location corresponding to the actual incision made during the CEA surgery. The m10 model was constructed by reducing the circumference of the vessel lumen along the incision line. The negative values of the virtual patch p10 width in Table 1 indicate this. Note that Table 1 shows the increments of the vessel perimeter after implantation, while the width of the patch itself before implantation should be slightly larger.

\section{Flow simulation}

The flow velocity $\mathrm{U}$ and pressure $\mathrm{p}$ in the constructed geometrical models were described through the three-dimensional unsteady Navier-Stokes equations for the viscous incompressible fluid [34]:

$$
\begin{aligned}
& \rho\left(\frac{\partial U}{\partial t}+(U \cdot \nabla) U\right)=-\nabla p+\nabla \tau, \\
& \nabla U=0
\end{aligned}
$$

at constant density $\rho=1050 \mathrm{~kg} / \mathrm{m}^{3}$ and dynamic viscosity $\mu=3.675 \cdot 10^{-3} \mathrm{~Pa} \cdot \mathrm{s}$, here $\tau$ is the shear stress tensor. The no-slip boundary condition was set for $U$ on the side surface of the flow region, and the parallel flow conditions were set on the inlet and outlets. The flow area boundaries were considered to be rigid. The initial velocity value was set to a constant of $0.15 \mathrm{~m} / \mathrm{s}$.

A periodically changing pressure difference was set as the boundary conditions for $p$ at the inlet and outlets. It created a periodic flow with parameters corresponding to the postoperative data of the patient's UDV:

$\cdot \mathrm{T}=1.06 \mathrm{~s}-$ a period of the cardiac cycle;

$\cdot \mathrm{Q}=6.9 \mathrm{ml} / \mathrm{s}$ volumetric flow rate through CCA;

$\cdot r=1.72$ the ratio of the volumetric flow rate through ICA to the volumetric flow rate through ECA.

The method for constructing boundary conditions was as follows. First, a preliminary CFD simulation was performed where the zero pressure was set at both outlets and a suitable pressure curve was built at the inlet stepwise with a time step of $10 \mathrm{~ms}$. During this simulation, the pressure increments (decrements) at each time step were selected manually. This made it possible to obtain an inlet velocity curve (Fig. 4 (a)) corresponding to the envelope of the UDV spectrum in the patient's CCA. Based on these simulation results, the ratio $r$ was calculated. It turned out to be different from the target value of 1.72. Therefore, the time-dependent pressure was set at the outlets instead of zero to adjust the r-value. Specifically, the same pressure curve was set at the ICA outlet as at the CCA inlet. It was only reduced in magnitude with a coefficient of $k=0.1$ and a small phase lag was set. The same pressure curve was set at the ECA outlet as at the ICA one. It was only inverted relative to the abscissa axis. Thus, the ECA flow decreased, and the 
ICA flow increased. The CCA flow remained practically unchanged. After that, using another series of auxiliary simulations, the value of the parameter $k$ (and the pressure curve shape, if needed) were adjusted so that the $\mathrm{Q}$ and $\mathrm{r}$ quantities became close to their target values.

\section{Numerical calculations and post-processing}

The flow simulation was performed in OpenFoam with the Finite Volume method using the PISO algorithm $[34,35]$. Along with commercial software such as Ansys Fluent, OpenFoam is a common tool for performing hydrodynamic calculations and modeling flow in blood vessels (see for example, $[4,18$, $36]$ ). In the preliminary numerical calculations described in section 2.3 , rough meshes were used to obtain the flow corresponding to the UDV data. After obtaining satisfactory results of preliminary calculations, the final calculations were made on fine meshes. The numerical calculations resulted in the dynamic fields of pressure, velocity, and velocity gradient in the flow domain for several cardiac cycles with a time sampling of $10^{-2} \mathrm{~s}$. The information about various flow parameters and their derived characteristics was extracted from the calculation results by postprocessing performed in ParaView [37].

To verify these results, a mesh independence study was conducted. It was found that the results do not significantly change when using meshes with the number of nodes more than $5 \cdot 10^{5}$. The mesh cell size on the side surface was set equal to half the cell size inside the computational domain. This was done to improve the accuracy of the calculation of hemodynamic indices which are expressed through the velocity gradient on the vessel wall. The stabilization of pulse oscillations was also studied. As it turns, the process of pulse oscillations can be considered as stabilized one starting with the second cardiac cycle (see also [38]). In this regard, it is further considered that the time $t=0$ corresponds to the beginning of the systolic phase of the second cardiac cycle.

\section{Wall Shear Stress and hemodynamic indices}

The WSS index was calculated as the tangential component $t_{w}$ of the shear stress tensor $\tau$ on the vessel wall. The TAWSS, OSI, and RRT indices were calculated with the formulas (2) through the averaged value of $t_{w}$ over the one cardiac cycle [24]:

TAWSS $=\frac{1}{T} \int_{0}^{T} \tau_{w} d t, \quad O S I=\frac{1}{2} \cdot\left(1-\frac{\left|\int_{0}^{T} \tau_{w} d t\right|}{\int_{0}^{T}\left|\tau_{w}\right| d t}\right), \quad R R T=\frac{T}{(1-2 \cdot O S I) \cdot \int_{0}^{T}\left|\tau_{w}\right| d t}$.

Here $T$ is the duration of the cardiac cycle and $\left|\tau_{w}\right|$ is the Euclidean norm of the vector $\tau_{w}$. For quantitative comparison of the indices in a certain zone $\sigma$ on the vessel wall their average values were calculated using the formulas: 
$R R T_{\sigma}=\frac{1}{s} \int_{\sigma} R R T d \sigma, \quad$ OSI $_{\sigma}=\frac{1}{s} \int_{\sigma}$ OSId $\sigma, \quad$ TAWSS $_{\sigma}=\frac{1}{s} \int_{\sigma}|T A W S S| d \sigma$,

where $S$ is the area of the zone $\sigma$. Dimensionless mean I_RRT and logarithmic maximum M_RRT values of the RRT index for the zone $\sigma$ were also used, calculated by formulas:

$$
\begin{aligned}
& I_{-} R R T=T A W S S_{C C A} \cdot R R T_{\sigma}, \\
& M_{-} R R T=\max _{\sigma}\left(\ln \left(R R T \cdot T A W S S_{C C A}+1\right)\right) .
\end{aligned}
$$

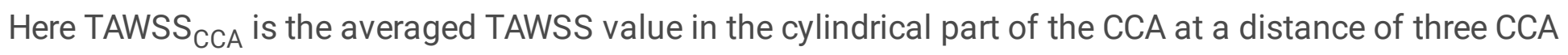
radii from the vessel bifurcation [24].

To assess the pathological values of the RRT index, the estimates TAWSS $<0.4 \mathrm{~Pa}$ and OSI $>0.3$, given in $[9,28]$, were used. In this case, formula (2) gives the corresponding critical value RRT $=6.25 \mathrm{~Pa}^{-1}$. Further, RRT values will be considered pathological if they are greater than the critical ones.

\section{Results And Discussion}

\section{Results for the base model}

One performed a qualitative analysis of the velocity field and hemodynamic indices calculation results for the base model m0. Fig. 4 shows some visualizations for $T=1.06 \mathrm{~s}, \mathrm{Q}=6.9 \mathrm{ml} / \mathrm{s}, \mathrm{r}=1.72$. Fig. 4 (a) shows the plot of the velocity magnitude in the center of the CCA proximal cross-section. Respectively, Figs. $4(b-d)$ show the streamlines at time points $t=0.06 \mathrm{~s}, 0.14 \mathrm{~s}, 0.6 \mathrm{~s}$, which are marked with dots in Fig. 4 (a). The color changes along a streamline reflect the velocity of the blood particles following the provided scale.

One also studied the influence of pulse frequency and magnitude on the distribution of hemodynamic indices on the vessel wall in the area of the atherosclerotic lesion. Table 2 shows the values of $T, Q$, and $r$ for the four simulations, indicated by the letters a-d.

Figures 5 (a-d) show the RRT level lines on a selected area of the vessel wall, corresponding to the same name sets of the parameters from Table 2. Level lines marked with 0 corresponding to the critical value $\mathrm{RRT}=6.25 \mathrm{~Pa}-1$, mentioned in section 2.5. Level lines $1-3$ correspond to RRT values 2,4 , and 8 times higher, respectively than the critical value. The color shows the $\ln (R R T+1)$ value between 0 and 8 according to the attached scale. Fig. 7 shows the selected area location on the vessel wall. Figures 5 (a-d) demonstrate that there is no significant qualitative change in the distribution of the RRT index when the flow parameters (given in Table 2) vary.

Figure 6 shows a slightly enlarged area of local maximums of the RRT index from Fig. 5 (a) in combination with a phase portrait of the TAWSS vector field. The arrows indicate the TAWSS vector field 
local direction, the color reflects the $\ln (\mathrm{RRT}+1)$ value in the interval $[0,8]$. Black dots are fixed points of the TAWSS field, bold lines are separatrices of two saddle points 2 and 4, and thin lines are regular trajectories.

For a comparative quantitative assessment of the RRT integral values, three zones $\sigma 1-\sigma 3$ with the same areas were selected (Fig. 6). The centers of these zones are the stationary points 1-3 of the TAWSS field. The average values of the indices calculated for each zone using the formulas (3) are shown in Table 3.

\section{Hemodynamic indices for $\mathrm{m} 0$-m10 models}

For m1-m10 models, we obtained the results of numerical calculations with flow parameters $\mathrm{Q}$ and $\mathrm{r}$ that differ by no more than $0.7 \%$ from the calculation result "a" for the base model (see Table 2 ). Changes in the geometric shape of the vessel caused by the implantation of a virtual patch lead to changes (within $10 \%$ ) in the calculated values of $Q$ and $r$ under unchanged boundary conditions. Therefore, described in section 2.3 , the auxiliary calculations were performed for each of the $\mathrm{m} 1-\mathrm{m} 10$ models to adjust the $k$ coefficient to meet the interval $[-0.7 \%, 0.7 \%]$ of changes in the parameters $\mathrm{Q}$ and $\mathrm{r}$.

To get comparative quantitative characteristics of hemodynamic indices, an area was selected in each model where both criteria TAWSS $<0.4 \mathrm{~Pa}$ and OSI $>0.3$ (see section 2.2) are simultaneously satisfied. These areas for all models consist of two zones z_1 and z_2. The first one contains stationary points 1,2, and the second one contains stationary points 3,4 of the TAWSS field. Fig. 7 shows these zones for the base model. Calculated using formulas (4) for each model, the values of the I_RRT and M_RRT indices in zones z_1 and z_2, are shown in Table 4. The table rows show the I_RRT and M_RRT values for the m0$\mathrm{m} 10$ models as a percentage of the corresponding values for the base $\mathrm{m} 0$ model.

\section{Discussion}

\section{Construction of geometric models}

The creation of a vessel geometric model based on its segmentation is considered in several works [12, $31,39,40]$. Our task was somewhat different, viz. to reconstruct the segmentation of the original healthy vessel from CTA data of the affected vessel, as was done in [41]. In undamaged areas of the vessel (Fig. 1 (b)), the boundaries of its lumen coincide with its inner wall, and segmentation in SimVascular can be performed automatically. But there is no such coincidence in the areas of the atherosclerotic lesion (Fig. 1 (c)), so each contour was built using manual correction followed by Fourier smoothing. For example, in Fig. 1 (c), the right part of the contour was built along the outer border of the x-ray contrast calcified plaque. Besides, due to the limited resolution of the equipment, the boundary of the vessel lumen is blurred, therefore, there is some scaling uncertainty in its recognition. Therefore, the areas of some recognized cross-sections were compared with the areas of the same cross-sections obtained by the UDV means, a correction factor was calculated, and then all the contours were scaled according to this factor. As a result, the CCA-ICA and ECA segmentations were obtained (Fig. 1 (d)). They were used to build the base model m0 (Fig. 1 (e)). 


\section{Flow simulation}

The flow simulation was performed under the assumption that blood is a Newtonian fluid. This is generally incorrect, but it has long been established that non-Newtonian rheology manifests itself mainly in small-diameter vessels and capillaries $[42,43]$. Therefore, Newtonian rheology is usually used to simulate the flow in the carotid artery [22, 28, 44], some works use other rheological models [27, 30, 47].

The assumption of a rigid vessel wall was accepted in this work for three reasons. Firstly, a healthy carotid artery is located in a bed of elastic and compliant structures of connective and adipose tissue, where the wall moves within. With the calcified atherosclerotic plaque appearance, the pulse movements of the vessel wall in its zone cease due to a lack of flexibility. Removal of the plaque and subsequent positioning of a patch in its place, however, does not restore the flexibility of the vessel wall in full, since the pliable surrounding structures are replaced by rigid connective tissue due to surgical trauma. Note that the lack of movement of the vascular wall in response to changes in pressure inside the vessel from a medical point of view is the most unfavorable hemodynamic option. The one which was chosen in this work as the model with the worst prognosis. Secondly, the studied areas of the vessel are much shorter than the length of the pulse wave of pressure. Therefore, in the case of an elastic wall, it can be assumed that they expand and contract almost synchronously. Hence, if the amplitude of the vessel diameter pulsations is estimated at $5 \%$, then the amplitude of fluctuations in the peak WSS value will not exceed $15 \%$ [44]. However, for integral indices (2), this peak systolic value is averaged over the entire cardiac cycle period and its effect on indices will be several times less. Finally, the introduction of additional input data required for FSI (Fluid-Solid Interaction) calculations, such as wall thickness and its mechanical properties, require their accurate assessment. Otherwise, the assumed errors in these data may introduce uncontrolled uncertainty in the results of calculations [44]. For these reasons, the assumption of wall stiffness is quite common for the carotid artery $[16,30,36,45]$.

\section{Results for model m0}

One knows that the flow in carotid bifurcation has a complex structure. It always contains time-varying recirculation and stagnation zones $[25,46,47]$. In our case, the qualitative behavior of the streamlines shown in Fig. 4 (b-d) corresponds to the published results.

To determine the probable positions of atherosclerotic plaque initiation zones and their dependence on blood flow parameters, the distributions of hemodynamic indices were obtained (Fig. 5) for four simulation variants (Table 2.). Figs. 5(a-d) demonstrate that the location of zones with the high values of RRT practically does not depend on changes in the parameters $\mathrm{T}$ and $\mathrm{Q}$.

Figure 6 shows the phase portrait of the TAWSS vector field corresponding to the set of parameters "a" in Table 2. In the area under consideration, the TAWSS field has four stationary points marked in Figure 6 with numbers 1-4. Points 1 and 3 are stable nodes, points 2 and 4 are saddle points (unstable) [48]. The arrows in Figure 6 correspond to the direction of shear stress action on endothelial cells. The phase trajectories of the TAWSS field correspond to the transport pathways of blood components and 
chemicals along the vessel wall [19]. Also, the calculations carried out for the sets of parameters b-d from Table 2, showed that the topological structure of the TAWSS vector field in the considered region is identical for all four variants of a-d. This fact, together with the results shown in Fig. 5, allows us to conclude that changes in the flow parameters have a smaller impact on the risk zones configuration compared to changes in the geometric shape of the vessel.

Table 3 shows that the zones $\sigma 1-\sigma 3$ are arranged in ascending order of their numbering in the context of indices values deterioration. Note that the location of the real atherosclerotic plaque in Fig. 1 (a), correlates with the location of the risk zones $\sigma 1-\sigma 3$ in Fig. 6. Plaque emergence changes local hemodynamics. It leads to the spread of pathological areas and the plaque's further growth $[49,50]$. Therefore, it can be assumed that the formation of plaque in a healthy $\mathrm{m} 0$ vessel began from stationary points of the TAWSS field. According to Table 3, the highest probability of pathological events refers to the zone $\sigma 3$ (other things being equal).

\section{Comparative analysis of results for $\mathrm{m} 0-\mathrm{m} 10$ models}

Comparative results of I_RRT and M_RRT indices calculations in zones z_1 and z_2 for all models are shown in Table 4. Zones z_1 and z_2 are the zones of the most probable occurrence of atherosclerotic events, higher values of indices in them mean greater risks of restenosis. According to Table 1, the patches p1-p4 have an approximately constant width along their entire length, excepting the ends.

Patches of this form are most often used by practicing surgeons, while the choice of patch width is left to them. In the literature, no rules have been found for choosing the patch width in a particular case, although some works (ex. [27, 28]) consider patches of different widths. The issue of choosing between implantation of the patch and suturing the incision without a patch is also discussed $[29,51]$. Table 4 shows that in terms of the risk of restenosis in each of the zones z_1 and z_2 among patches p1-p4, it is preferable to use $\mathrm{p} 3$. The preference for using a patch over directly suturing the incision in our case is confirmed by the data in Table 4 for the m10 model, which imitates this type of surgery.

In this work, in contrast to those mentioned above, patches of uneven width were also studied. As seen from Table 1, the $p 5$ and $p 6$ patches were derived from patch $p 3$ by narrowing and widening it in the proximal part, respectively. Both options lead to a deterioration of the index values in Table 4 .

The $p 7$ and $p 8$ patches were obtained from $p 2$. The $p 7$ patch is $p 2$ tapered at its proximal part. The $p 8$ patch is a $0.7 \mathrm{~cm}$ shortened $\mathrm{p} 2$, implanted into the incision, with incision primary suture in the proximal part with reducing the circumference of the vessel lumen by $0.1 \mathrm{~cm}$. In both cases, there is a slight improvement in the indices in the z_1 zone and deterioration in the z_2 zone compared to patch p2.

The $\mathrm{p} 9$ patch was obtained from $\mathrm{p} 4$ by narrowing it in the distal part and this does not lead to any significant changes in the values of indices in Table 4.

Thus, in our case, the best option is to choose the p3 patch, and the worst option is to suture the incision without patching. 


\section{Conclusion}

In this work, using the proposed method, the hemodynamic results of 10 virtual CEA surgeries with patches of various shapes were studied on the example of a reconstructed healthy artery of a particular patient. The purpose of patch implantation is to ensure that the vessel lumen is not narrowed as a result of the surgery, since suturing the incision without a patch can reduce the circumference of the vessel lumen by $4-5 \mathrm{~mm}$, which adversely affects blood flow. On the other hand, too wide a patch creates aneurysm-like deformation of the ICA mouth, which is not optimal due to the formation of a large recirculation zone. In this case, it was found that the width of the implanted patch, approximately equal to $3 \mathrm{~mm}$, provides an optimal hemodynamic result. Deviations from this median value, both upward and downward, worsen hemodynamics, and the suture without a patch gives the worst of the considered results. Some forms of patches of uneven width were also considered, but in this case, none of them gave a positive result, although there are carotid bifurcations for which a patch of just such a shape would be optimal. The proposed technique in this case can help in the experimental selection of a suitable patch shape.

\section{Declarations}

Conclusion of the local ethics committee No. 5 dated December 6, 2020. The local ethics committee was held at the Amur State Medical Academy in Blagoveshchensk, Russia.

\section{DECLARATION OF COMPETING INTEREST}

There is no conflict of interests.

\section{FUNDING INFORMATION}

This research was funded by the Basic Research Topic of Research Institute for Complex Issues of Cardiovascular Diseases №0546-2019-0002 "Pathogenetic basis for the development of cardiovascular implants from biocompatible materials using the patient-oriented approach, mathematical modeling, tissue engineering, and genomic predictors".

\section{Abbreviations}

$\mathrm{CEA}=$ carotid endarterectomy

CFD = Computational Fluid Dynamics

WSS $=$ Wall Shear Stress

OSI = Oscillatory Shear Index 
TAWSS $=$ Time Averaged WSS

RRT = Relative Residence Time

CTA = Computed Tomography Angiography

UDV = Ultrasound Doppler Velocimetry

$\mathrm{CCA}=$ Common Carotid Artery

ICA = Internal Carotid Artery

ECA $=$ External Carotid Artery

\section{References}

1. Saha SP, Saha S, Vyas KS. Carotid Endarterectomy: Current Concepts and Practice Patterns. Int J Angiol. 2015 Sep;24(3):223-35. doi: 10.1055/s-0035-1558645. Epub 2015 Aug 14. PMID: 26417192; PMCID: PMC4572020.

2. Fairhead JF, Rothwell PM. The need for urgency in identification and treatment of symptomatic carotid stenosis is already established. Cerebrovasc Dis. 2005;19(6):355-8. doi: 10.1159/000085201. Epub 2005 Apr 15. PMID: 15838162.

3. Zhong L, Zhang JM, Su B, Tan RS, Allen JC, Kassab GS. Application of Patient-SpecifiC Computational Fluid Dynamics in Coronary and Intra-Cardiac Flow Simulations: Challenges and Opportunities. Front Physiol. 2018 Jun 26;9:742. doi: 10.3389/fphys.2018.00742. PMID: 29997520; PMCID: PMC6028770.

4. Gijsen F, Katagiri Y, Barlis P, Bourantas C, Collet C, Coskun U, Daemen J, Dijkstra J, Edelman E, Evans P, van der Heiden K, Hose R, Koo BK, Krams R, Marsden A, Migliavacca F, Onuma Y, Ooi A, Poon E, Samady H, Stone P, Takahashi K, Tang D, Thondapu V, Tenekecioglu E, Timmins L, Torii R, Wentzel J, Serruys $\mathrm{P}$. Expert recommendations on the assessment of wall shear stress in human coronary arteries: existing methodologies, technical considerations, and clinical applications. Eur Heart J. 2019 Nov 1;40(41):3421-3433. doi: 10.1093/eurheartj/ehz551. PMID: 31566246; PMCID: PMC6823616.

5. Caro CG, Fitz-Gerald JM, Schroter RC. Atheroma and arterial wall shear. Observation, correlation and proposal of a shear dependent mass transfer mechanism for atherogenesis. Proc R Soc Lond B Biol Sci. 1971 Feb 16;177(1046):109-59. doi: 10.1098/rspb.1971.0019. PMID: 4396262.

6. Zarins CK, Giddens DP, Bharadvaj BK, Sottiurai VS, Mabon RF, Glagov S. Carotid bifurcation atherosclerosis. Quantitative correlation of plaque localization with flow velocity profiles and wall shear stress. Circ Res. 1983 Oct;53(4):502-14. doi: 10.1161/01.res.53.4.502. PMID: 6627609.

7. Irace C, Cortese C, Fiaschi E, Carallo C, Farinaro E, Gnasso A. Wall shear stress is associated with intima-media thickness and carotid atherosclerosis in subjects at low coronary heart disease risk. 
Stroke. 2004 Feb;35(2):464-8. doi: 10.1161/01.STR.0000111597.34179.47. Epub 2004 Jan 15. PMID: 14726547.

8. Ku DN, Giddens DP, Zarins CK, Glagov S. Pulsatile flow and atherosclerosis in the human carotid bifurcation. Positive correlation between plaque location and low oscillating shear stress. Arteriosclerosis. 1985 May-Jun;5(3):293-302. doi: 10.1161/01 atv.5.3.293. PMID: 3994585.

9. Malek AM, Alper SL, Izumo S. Hemodynamic shear stress and its role in atherosclerosis. JAMA. 1999 Dec 1;282(21):2035-42. doi: 10.1001/jama.282.21.2035. PMID: 10591386.

10. Lei M, Kleinstreuer C, Archie JP Jr. Geometric design improvements for femoral graft-artery junctions mitigating restenosis. J Biomech. 1996 Dec;29(12):1605-14. PMID: 8945659.

11. Loth F, Jones SA, Zarins CK, Giddens DP, Nassar RF, Glagov S, Bassiouny HS. Relative contribution of wall shear stress and injury in experimental intimal thickening at PTFE end-to-side arterial anastomoses. J Biomech Eng. 2002 Feb;124(1):44-51. doi: 10.1115/1.1428554. PMID: 11871604.

12. LaDisa JF Jr, Bowers M, Harmann L, Prost R, Doppalapudi AV, Mohyuddin T, Zaidat O, Migrino RQ. Time-efficient patient-specific quantification of regional carotid artery fluid dynamics and spatial correlation with plaque burden. Med Phys. 2010 Feb;37(2):784-92. doi: 10.1118/1.3292631. PMID: 20229888; PMCID: PMC2826384.

13. J. Lantz, J. Rennerm, M. Karlsson, Wall shear stress in a subject specific human aorta - influence of fluid-structure interaction, International Journal of Applied Mechanics 03(04) (2011)759-778. https://doi.org/10.1142/S1758825111001226

14. Shintani Y, lino K, Yamamoto Y, Kato H, Takemura H, Kiwata T. Analysis of Computational Fluid Dynamics and Particle Image Velocimetry Models of Distal-End Side-to-Side and End-to-Side Anastomoses for Coronary Artery Bypass Grafting in a Pulsatile Flow. Circ J. 2017 Dec 25;82(1):110-117. doi: 10.1253/circj.CJ-17-0381. Epub 2017 Aug 19. PMID: 28824030.

15. Lee SW, Antiga L, Spence JD, Steinman DA. Geometry of the carotid bifurcation predicts its exposure to disturbed flow. Stroke. 2008 Aug;39(8):2341-7. doi: 10.1161/STROKEAHA.107.510644. Epub 2008 Jun 12. PMID: 18556585.

16. Domanin M, Buora A, Scardulla F, Guerciotti B, Forzenigo L, Biondetti P, Vergara C. Computational Fluid-Dynamic Analysis after Carotid Endarterectomy: Patch Graft versus Direct Suture Closure. Ann Vasc Surg. 2017 Oct;44:325-335. doi: 10.1016/j.avsg.2017.04.016. Epub 2017 May 4. PMID: 28479438.

17. Himburg HA, Grzybowski DM, Hazel AL, LaMack JA, Li XM, Friedman MH. Spatial comparison between wall shear stress measures and porcine arterial endothelial permeability. Am J Physiol Heart Circ Physiol. 2004 May;286(5):H1916-22. doi: 10.1152/ajpheart.00897.2003. Epub 2004 Jan 8. PMID: 14715506.

18. E. Soudah, J. Casacuberta, P.J. Gamez-Montero, G. Raush, R. Castilla, Estimation of wall shear stress using 4D flow cardiovascular MRI and computational fluid dynamics, Journal of mechanics in medicine and biology 17(3) (2017) 1-16. https://doi.org/10.1142/S0219519417500464 
19. Arzani A, Shadden SC. Wall shear stress fixed points in cardiovascular fluid mechanics. J Biomech. 2018 May 17;73:145-152. doi: 10.1016/j.jbiomech.2018.03.034. Epub 2018 Mar 29. PMID: 29625775.

20. Longest PW, Kleinstreuer C, Deanda A. Numerical simulation of wall shear stress and particle-based hemodynamic parameters in pre-cuffed and streamlined end-to-side anastomoses. Ann Biomed Eng. 2005 Dec;33(12):1752-66. doi: 10.1007/s10439-005-7784-2. PMID: 16389524.

21. P. Di Achille, G. Tellides, C.A. Figueroa, J.D. Humphrey, A haemodynamic predictor of intraluminal thrombus formation in abdominal aortic aneurysms, Proceedings of The Royal Society A Mathematical Physical and Engineering 470, (2014) 20140163. https://doi.org/10.1098/rspa.2014.0163

22. Geers AJ, Morales HG, Larrabide I, Butakoff C, Bijlenga P, Frangi AF. Wall shear stress at the initiation site of cerebral aneurysms. Biomech Model Mechanobiol. 2017 Feb;16(1):97-115. doi: 10.1007/s10237-016-0804-3. Epub 2016 Jul 20. PMID: 27440126.

23. Friedman MH, Deters OJ. Correlation among shear rate measures in vascular flows. J Biomech Eng. 1987 Feb;109(1):25-6. doi: 10.1115/1.3138637. PMID: 3560875.

24. Lee SW, Antiga L, Steinman DA. Correlations among indicators of disturbed flow at the normal carotid bifurcation. J Biomech Eng. 2009 Jun;131(6):061013. doi: 10.1115/1.3127252. PMID: 19449967.

25. Hayase H, Tokunaga K, Nakayama T, Sugiu K, Nishida A, Arimitsu S, Hishikawa T, Ono S, Ohta M, Date I. Computational fluid dynamics of carotid arteries after carotid endarterectomy or carotid artery stenting based on postoperative patient-specific computed tomography angiography and ultrasound flow data. Neurosurgery. 2011 Apr;68(4):1096-101; discussion 1101. doi:

10.1227/NEU.0b013e318208f1a0. PMID: 21221041.

26. Harrison GJ, Brennan JA, Naik JB, Vallabhaneni SR, Fisher RK. Patch variability following carotid endarterectomy: a survey of Great Britain and Ireland. Ann R Coll Surg Engl. 2012 Sep;94(6):411-5. doi: 10.1308/003588412X13373405385494. PMID: 22943331; PMCID: PMC3954322.

27. Kamenskiy AV, Pipinos II, Dzenis YA, Gupta PK, Jaffar Kazmi SA, Mactaggart JN. A mathematical evaluation of hemodynamic parameters after carotid eversion and conventional patch angioplasty. Am J Physiol Heart Circ Physiol. 2013 Sep 1;305(5):H716-24. doi: 10.1152/ajpheart.00034.2013. Epub 2013 Jun 28. PMID: 23812386; PMCID: PMC3761324.

28. Harrison GJ, How TV, Poole RJ, Brennan JA, Naik JB, Vallabhaneni SR, Fisher RK. Closure technique after carotid endarterectomy influences local hemodynamics. J Vasc Surg. 2014 Aug;60(2):418-27. doi: 10.1016/j.jvs.2014.01.069. Epub 2014 Mar 20. PMID: 24657293.

29. Guerciotti B, Vergara C, Azzimonti L, Forzenigo L, Buora A, Biondetti P, Domanin M. Computational study of the fluid-dynamics in carotids before and after endarterectomy. J Biomech. 2016 Jan 4;49(1):26-38. doi: 10.1016/j.jbiomech.2015.11.009. Epub 2015 Nov 18. PMID: 26617369.

30. Gharahi H, Zambrano BA, Zhu DC, DeMarco JK, Baek S. Computational fluid dynamic simulation of human carotid artery bifurcation based on anatomy and volumetric blood flow rate measured with 
magnetic resonance imaging. Int J Adv Eng Sci Appl Math. 2016 Mar;8(1):40-60. doi: 10.1007/s12572-016-0161-6. Epub 2016 Feb 2. PMID: 27546999; PMCID: PMC4987097.

31. Updegrove A, Wilson NM, Merkow J, Lan H, Marsden AL, Shadden SC. SimVascular: An Open Source Pipeline for Cardiovascular Simulation. Ann Biomed Eng. 2017 Mar;45(3):525-541. doi: 10.1007/s10439-016-1762-8. Epub 2016 Dec 8. PMID: 27933407; PMCID: PMC6546171.

32. Salomé platform component model for numerical simulation, COMPSAC 07: Proceeding of the 31st Annual International Computer Software and Applications Conference Washington DC USA IEEE Computer Society (2007) 553-564.

33. The Open Source CFD Toolbox User Guide OpenCFD Limited (2013).

34. J.H. Ferziger, M. Peric, Computational Methods for Fluid Dynamics, Springer Berlin Heidelberg 3rd Ed. (2001) $423 \mathrm{p}$.

35. R.I. Issa, Solution of the implicitly discretised fluid flow equations by operator-splitting, Journal of Computational Physics 62(1) (1985) 40-65. https://doi.org/10.1016/0021-9991(86)90099-9

36. Arzani A, Gambaruto AM, Chen G, Shadden SC. Wall shear stress exposure time: a Lagrangian measure of near-wall stagnation and concentration in cardiovascular flows. Biomech Model Mechanobiol. 2017 Jun;16(3):787-803. doi: 10.1007/s10237-016-0853-7. Epub 2016 Nov 17. PMID: 27858174.

37. U. Ayachit, The ParaView Guide: A Parallel Visualization Application Kitware ISBN 978-1930934306 (2015).

38. V.G. Borisov, Y.N. Zakharov, Y.I. Shokin, et. al., Numerical Method for Predicting Hemodynamic Effects in Vascular Prostheses, Numer. Analys. Appl. 12 (2019) 326-337.

39. Steinman DA. Image-based computational fluid dynamics modeling in realistic arterial geometries. Ann Biomed Eng. 2002 Apr;30(4):483-97. doi: 10.1114/1.1467679. PMID: 12086000.

40. Moccia S, De Momi E, El Hadji S, Mattos LS. Blood vessel segmentation algorithms - Review of methods, datasets and evaluation metrics. Comput Methods Programs Biomed. 2018 May;158:7191. doi: 10.1016/j.cmpb.2018.02.001. Epub 2018 Feb 10. PMID: 29544791.

41. Li X, Sun B, Zhao H, Ge X, Liang F, Li X, Xu J, Liu X. Retrospective Study of Hemodynamic Changes Before and After Carotid Stenosis Formation by Vessel Surface Repairing. Sci Rep. 2018 Apr 3;8(1):5493. doi: 10.1038/s41598-018-23842-0. PMID: 29615730; PMCID: PMC5883012.

42. C.G. Caro, T.J. Pedley, R.S. Schroter, W.A. Seed, The Mechanics of the Circulation, New York: Oxford Univ. Press (1978) 527p.

43. Cho YI, Kensey KR. Effects of the non-Newtonian viscosity of blood on flows in a diseased arterial vessel. Part 1: Steady flows. Biorheology. 1991;28(3-4):241-62. doi: 10.3233/bir-1991-283-415. PMID: 1932716.

44. D.A. Steinman, Assumptions in modelling of large artery hemodynamics. In Modeling of Physiological Flows, Springer-Verlag Italia (2012) 1-19. 
45. Zhang D, Xu P, Qiao H, Liu X, Luo L, Huang W, Zhang H, Shi C. Carotid DSA based CFD simulation in assessing the patient with asymptomatic carotid stenosis: a preliminary study. Biomed Eng Online. 2018 Mar 12;17(1):31. doi: 10.1186/s12938-018-0465-9. PMID: 29530025; PMCID: PMC5848462.

46. Hoskins PR, Hardman D. Three-dimensional imaging and computational modelling for estimation of wall stresses in arteries. Br J Radiol. 2009 Jan;82 Spec No 1:S3-17. doi: 10.1259/bjr/96847348. PMID: 20348533.

47. Morbiducci U, Gallo D, Massai D, Ponzini R, Deriu MA, Antiga L, Redaelli A, Montevecchi FM. On the importance of blood rheology for bulk flow in hemodynamic models of the carotid bifurcation. $J$ Biomech. 2011 Sep 2;44(13):2427-38. doi: 10.1016/j.jbiomech.2011.06.028. Epub 2011 Jul 12. PMID: 21752380.

48. J. Guckenheimer, Nonlinear oscillations, dynamical systems, and bifurcations of vector fields, Springer-Verlag New York Inc. Corr. 6th printing (2002) 462 p.

49. Arzani A. Coronary artery plaque growth: A two-way coupled shear stress-driven model. Int J Numer Method Biomed Eng. 2020 Jan;36(1):e3293. doi: 10.1002/cnm.3293. Epub 2019 Dec 9. PMID: 31820589.

50. Wu WT, Jamiolkowski MA, Wagner WR, Aubry N, Massoudi M, Antaki JF. Multi-Constituent Simulation of Thrombus Deposition. Sci Rep. 2017 Feb 20;7:42720. doi: 10.1038/srep42720. PMID: $28218279 ;$ PMCID: PMC5316946.

51. Avrahami I, Raz D, Bash O. Biomechanical Aspects of Closing Approaches in Postcarotid Endarterectomy. Comput Math Methods Med. 2018 Oct 28;2018:4517652. doi: 10.1155/2018/4517652. PMID: 30510592; PMCID: PMC6230422.

\section{Tables}

Table 1. Width values $(\mathrm{cm})$ of p1-p10 patches along the incision line. 


\begin{tabular}{|lllllllllll|}
\hline $\begin{array}{l}\text { Distance along the } \\
\text { incision }(\mathrm{cm})\end{array}$ & $\mathrm{p} 1$ & $\mathrm{p} 2$ & $\mathrm{p} 3$ & $\mathrm{p} 4$ & $\mathrm{p} 5$ & $\mathrm{p} 6$ & $\mathrm{p} 7$ & $\mathrm{p} 8$ & $\mathrm{p} 9$ & $\mathrm{p} 10$ \\
\hline .00 & 0.00 & 0.00 & 0.00 & 0.00 & 0.00 & 0.00 & 0.00 & 0.00 & 0.00 & -0.00 \\
0.29 & 0.12 & 0.16 & 0.19 & 0.34 & 0.13 & 0.35 & 0.05 & -0.10 & 0.34 & -0.26 \\
0.72 & 0.14 & 0.21 & 0.28 & 0.42 & 0.14 & 0.42 & 0.07 & 0.00 & 0.42 & -0.22 \\
1.16 & 0.13 & 0.20 & 0.27 & 0.39 & 0.25 & 0.29 & 0.18 & 0.19 & 0.39 & -0.22 \\
1.59 & 0.14 & 0.20 & 0.27 & 0.40 & 0.27 & 0.27 & 0.20 & 0.20 & 0.38 & -0.22 \\
2.02 & 0.15 & 0.22 & 0.29 & 0.43 & 0.29 & 0.29 & 0.22 & 0.22 & 0.33 & -0.24 \\
\hline 2.46 & 0.16 & 0.24 & 0.33 & 0.47 & 0.33 & 0.33 & 0.24 & 0.24 & 0.24 & -0.26 \\
\hline 2.89 & 0.15 & 0.23 & 0.32 & 0.45 & 0.32 & 0.32 & 0.23 & 0.23 & 0.23 & -0.26 \\
3.32 & 0.13 & 0.20 & 0.27 & 0.39 & 0.27 & 0.27 & 0.20 & 0.20 & 0.20 & -0.22 \\
3.61 & 0.12 & 0.17 & 0.23 & 0.34 & 0.23 & 0.23 & 0.17 & 0.17 & 0.15 & -0.14 \\
3.90 & 0.00 & 0.00 & 0.00 & 0.00 & 0.00 & 0.00 & 0.00 & 0.00 & 0.00 & 0.00 \\
\hline
\end{tabular}

Table 2. Flow parameters in simulation variants (a-d).

\begin{tabular}{|llll|}
\hline & $\mathrm{T}(\mathrm{s})$ & $\mathrm{Q}(\mathrm{ml} / \mathrm{s})$ & $\mathrm{r}$ \\
\hline $\mathrm{a}$ & 1.06 & 6.90 & 1.72 \\
$\mathrm{~b}$ & 1.06 & 6.00 & 1.78 \\
\hline $\mathrm{c}$ & 0.7 & 7.76 & 1.77 \\
$\mathrm{~d}$ & 0.8 & 10.65 & 1.72 \\
\hline
\end{tabular}

Table 3. Average index values in zones $\sigma 1-\sigma 3$.

\begin{tabular}{|lllll|}
\hline & $\mathrm{RRT}_{\sigma}\left(\mathrm{Pa}^{-1}\right)$ & $\ln \left(\mathrm{RRT}_{\sigma}+1\right)$ & $\mathrm{OSI}_{\sigma}$ & TAWSS $_{\sigma}(\mathrm{Pa})$ \\
\hline$\sigma_{1}$ & 287 & 5.66 & 0.409 & 0.047 \\
$\sigma_{2}$ & 739 & 6.61 & 0.428 & 0.049 \\
$\sigma_{3}$ & 974 & 6.88 & 0.453 & 0.031 \\
\hline
\end{tabular}

Table 4. Comparison of I_RRT and M_RRT indices for m0-m10 models 


\begin{tabular}{|lllll|}
\hline & I_RRT $_{1}$ & I_RRT $_{2}$ & M_RRT $_{1}$ & M_RRT $_{2}$ \\
\hline m0 & $100 \%$ & $100 \%$ & $100 \%$ & $100 \%$ \\
\hline m1 & $88.8 \%$ & $97.8 \%$ & $78.7 \%$ & $98.7 \%$ \\
\hline m2 & $78.6 \%$ & $94.7 \%$ & $75.9 \%$ & $95.8 \%$ \\
\hline m3 & $71.8 \%$ & $92.9 \%$ & $70.0 \%$ & $96.1 \%$ \\
\hline m4 & $76.1 \%$ & $96.5 \%$ & $74.3 \%$ & $96.8 \%$ \\
\hline m5 & $76.3 \%$ & $96.8 \%$ & $75.2 \%$ & $98.3 \%$ \\
\hline m6 & $75.4 \%$ & $98.1 \%$ & $82.5 \%$ & $97.3 \%$ \\
\hline m7 & $75.9 \%$ & $97.3 \%$ & $74.9 \%$ & $99.8 \%$ \\
\hline m8 & $75.7 \%$ & $97.0 \%$ & $74.2 \%$ & $99.9 \%$ \\
\hline m9 & $77.0 \%$ & $95.7 \%$ & $74.6 \%$ & $98.3 \%$ \\
\hline m10 & $82.0 \%$ & $110.0 \%$ & $81.9 \%$ & $105.9 \%$ \\
\hline
\end{tabular}

\section{Figures}

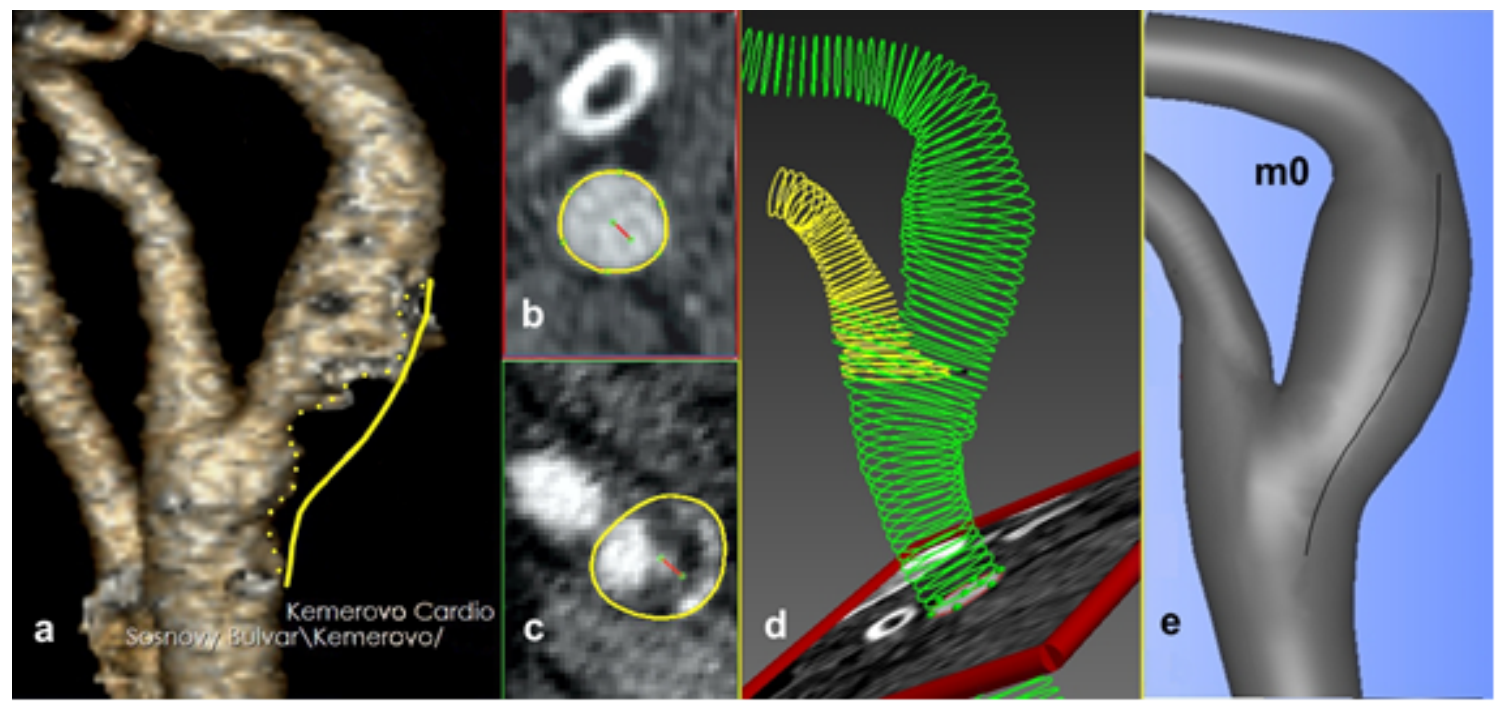

\section{Figure 1}

CTA image of the preoperative flow area in the carotid arteries (foreground vessel) (a). Segmentation contour in the healthy part of the CCA (b). Segmentation contour in the affected part of the ICA near the bifurcation (c). Segmentations of CCA-ICA and ECA (d). The geometric shape of the base model $\mathrm{m} 0$ with an incision line (e). 


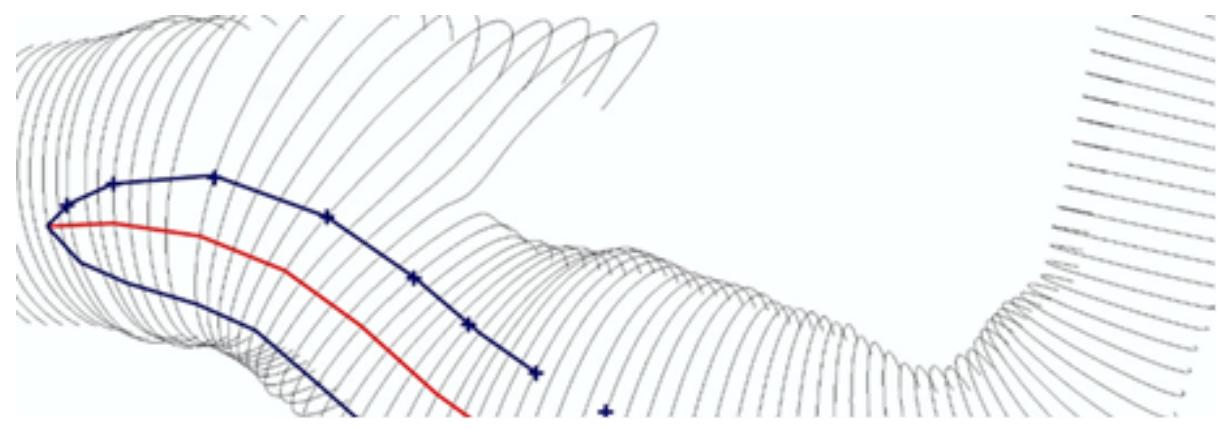

Figure 2

The process of visually constructing the patch shape on the OCA-ICA segmentation contours.

Figure 3

Shapes of patches and vessels after virtual CEA.

Figure 4

Velocity plot in the center of the CCA proximal cross-section (a). Streamlines at: $t=0.06 \mathrm{~s}(\mathrm{~b}), \mathrm{t}=0.14 \mathrm{~s}$ $(c), t=0.6 s(d)$.

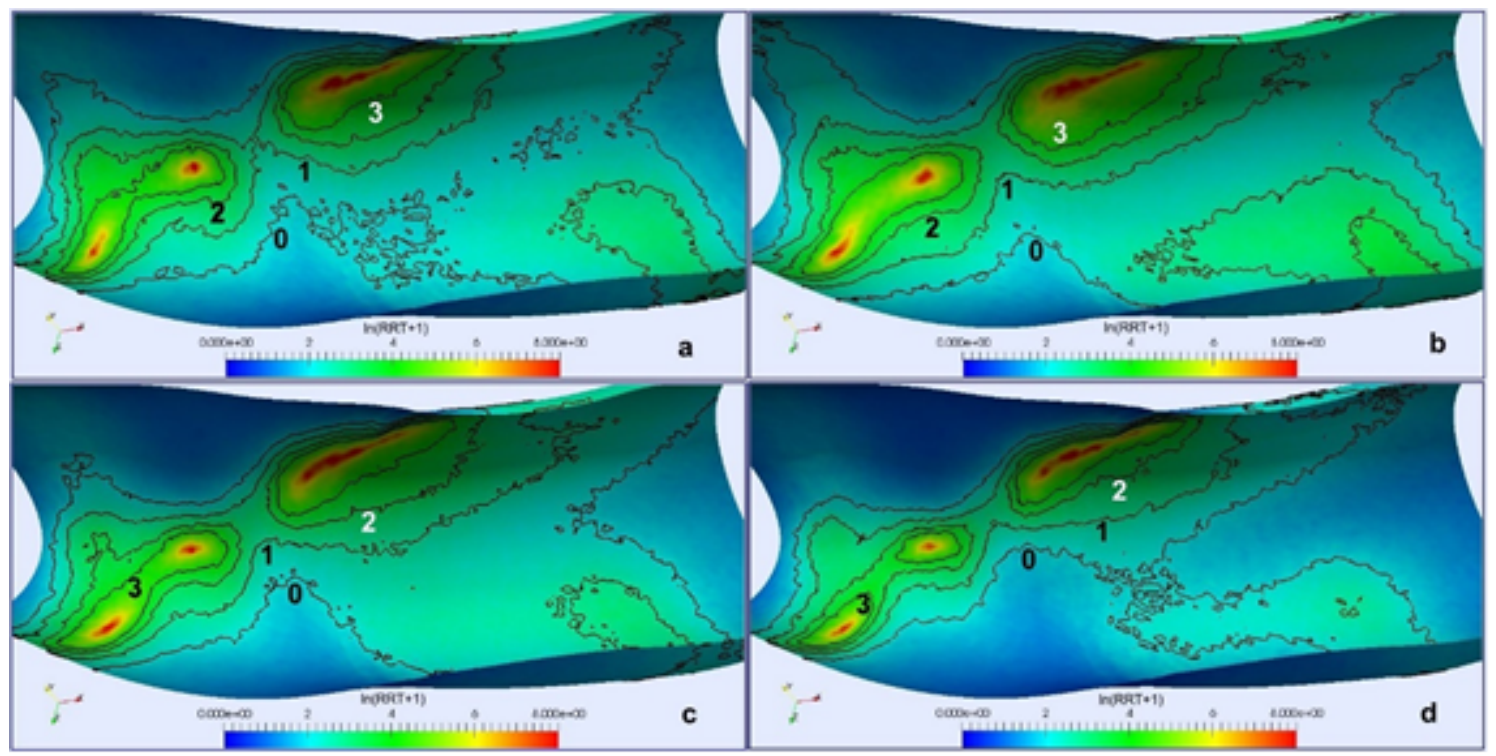

Figure 5 
RRT (Pa-1) index level lines and In(RRT+1) distribution for parameter sets (a-d) in Table 2.

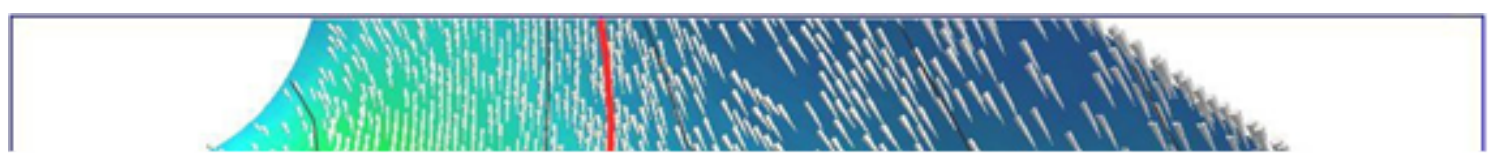

\section{Figure 6}

The topological structure of the TAWSS vector field in the vicinity of its stationary points (points 1-4).

\section{Figure 7}

Zones z_1 and z_2 and the TAWSS field stationary points 1-4 for the base model. 